\title{
A Novel Meshfree Strategy for a Viscous Wave Equation With Variable Coefficients
}

\author{
Fuzhang Wang ${ }^{1,2}$, Juan Zhang ${ }^{2 *},{\text { Imtiaz } \text { Ahmad }^{3} \text {, Aamir Farooq }}^{4}$ and Hijaz Ahmad ${ }^{5}$ \\ ${ }^{1}$ College of Mathematics and Statistics, Xuzhou University of Technology, Xuzhou, China, ${ }^{2}$ School of Computer Science and \\ Technology, Huaibei Normal University, Huaibei, China, ${ }^{3}$ Department of Mathematics, University of Swabi, Swabi, Pakistan, \\ ${ }^{4}$ Department of Mathematics, Abbottabad University of Science and Technology, Abbottabad, Pakistan, ${ }^{5}$ Section of \\ Mathematics, International Telematic University Uninettuno, Roma, Italy
}

OPEN ACCESS

Edited by:

Umberto Lucia,

Politecnico di Torino, Italy

Reviewed by:

Lihua Wang,

Tongji University, China

Aatish Anshuman,

Indian Institute of Technology

Bombay, India

Hanen Jrad,

National Engineering School of Sfax,

Tunisia

*Correspondence:

Juan Zhang

zj801106@163.com

Specialty section:

This article was submitted to Interdisciplinary Physics,

a section of the journal

Frontiers in Physics

Received: 28 April 2021 Accepted: 08 June 2021

Published: 02 July 2021

Citation:

Wang F, Zhang J, Ahmad I, Farooq A and Ahmad H (2021) A Novel Meshfree Strategy for a Viscous Wave Equation With Variable Coefficients.

Front. Phys. 9:701512.

doi: 10.3389/fphy.2021.701512
A one-step new general mesh free scheme, which is based on radial basis functions, is presented for a viscous wave equation with variable coefficients. By constructing a simple extended radial basis function, it can be directly applied to wave propagation by using the strong form-based mesh free collocation method. There is no need to deal with the timedependent variable particularly. Numerical results for a viscous wave equation with variable coefficients show that the proposed mesh free collocation method is simple with accurate solutions.

Keywords: radial basis functions, wave equations, numerical simulation, meshfree methods, variable

\section{INTRODUCTION}

The wave propagation exists in an interesting class of problems, such as the micro-scale heat transfer, seismic data acquisition and processing, etc. Acoustic wave modeling is an essential part of the technique of acoustic imaging [1]. Such problems have been widely investigated because of their realistic physical background. Related works can be found in [2] and references therein.

Since the governing equation of the problem considered is complex, and the corresponding physical domain or the boundary conditions are tangle some, the analytical solutions are almost inaccessible for most practical engineering problems [3-7]. The development of accurate and efficient numerical methods becomes an important research topic in computational physics.

The earliest numerical methods for wave equations include the finite difference method [8], the finite element method [9], the boundary element method [10] and the finite volume method [11]. It should be noted that most of the existed numerical methods are still based on the finite difference method (FDM) [12, 13], which lead to two-step finite difference approximations. More specifically, the finite difference method is used to deal with the time variable, and the rest procedures are finished by the other numerical methods. Coupled with the FDM, the radial basis function (RBF) collocation methods are widely-used to many problems thanks to their mesh free features. Akers et al. [14] investigated a radial basis function-finite differencing (RBF-FD) scheme which is applied to the initial value problem of the Benjamin-Ono equation. Bhardwaj and Kumar [15] used the FDM-based mesh less approach for the numerical solution of the nonlinear diffusion-wave equation. Based on the integrated radial basis functions and a finite difference scheme, the regularized long-wave equations are investigated in [16, 17]. Oruc [18] examined numerical solutions of ZakharovRubenchik system by using radial basis function finite difference (RBF-FD) mesh less method and an explicit Runge-Kutta method. The time discretization is accomplished by means of an implicit method based on the theta-weighted and finite difference methods, while the spatial discretization is described with the help of the finite difference scheme derived from the local radial basis function 
method. Recently, Lu et al [19] used the radial basis function mesh less method to solve the irregular region interface problem. Ranocha et al [20] created new classes of fully-discrete conservative methods for several nonlinear dispersive wave equations.

In order to simplify the conventional two-step solution process, Netuzhylov and Zilian [21] proposed a space-time mesh free collocation method for solving partial differential equations by a consistent discretization in both space and time. Motivated by this work, we aim to provide a truly mesh free method with one-step approximation, which is based on the RBFs, for a viscous wave equation with variable coefficients. Compared with the traditional FDM-based two-step methods, the newly-proposed one-step direct mesh free method is truly mesh free with easy implementation. The computational efficiency is excellent and the computational accuracy is mainly determined by numerical methods for wave field simulation.

The paper is organized as follows. In Section 2, formulation of the direct radial basis function with space-time Euclidean distance is briefly introduced. This is realized by considering time-dependent variable as normal time-independent variable. Thus, the time-dependent variable can be treated easily during the whole solution process. Section 3 presents the methodology for a viscous wave equation with variable coefficients under initial condition and boundary conditions. Numerical results with detailed discussions are presented for the accuracy of the proposed mesh free method in Section 4. Section 5 concludes this paper with some additional remarks.

\section{PROBLEM DESCRIPTION}

Let's consider a viscous wave equation on a bounded twodimensional domain $\Omega \subset R^{2}$

$$
L u \equiv \frac{\partial^{2} u}{\partial t^{2}}+\alpha \frac{\partial u}{\partial t}-\frac{\partial}{\partial t} \nabla \cdot(Q \nabla u)-\nabla \cdot(A \nabla u)=f(x, y, t), t>0
$$

where $\alpha$ is a nonnegative coefficient, $f$ is a source term, $Q=$ $\operatorname{diag}\left(q_{1}, q_{2}\right)$ and $A=\operatorname{diag}\left(a_{1}, a_{2}\right)$ are diagonal, nonnegative diffusion tensors with $q_{1}, q_{2}, a_{1}, a_{2}$ constant or variable coefficients. Solution to Eq. 1 requires the extra initial conditions

$$
\begin{aligned}
& u(x, y, 0)=f_{1}(x, y),(x, y) \in \Omega \\
& u_{t}(x, y, 0)=f_{2}(x, y),(x, y) \in \Omega
\end{aligned}
$$

and boundary conditions

$$
B u(x, y, t)=f_{3}(x, y, t),(x, y) \in \partial \Omega, t>0
$$

with $f_{1}(x, y), f_{2}(x, y)$ and $f_{3}(x, y, t)$ the given functions. $B$ is a boundary operator. One should seek for the numerical solution of unknown function $u(x, y, t)$.

A variety of numerical methods have been investigated for Eqs. 1-4. Most of them are based on the finite difference method, which lead to two-step methods. More specifically, the finite difference method is used to deal with the time domain, which will lead to elliptic-type problems. Then the rest work is done by the other numerical methods. In order to improve the perplexing two-step methods, we propose a direct one-step collocation method by using an extended RBFs in the following section.

\section{FORMULATION OF THE EXTENDED RADIAL BASIS FUNCTIONS}

The radial basis functions (RBFs) are extremely powerful tool for solving partial differential equations. It is "isotropic" for Euclidean spaces. The Kansa's method $[22,23]$ is one of the most famous RBFs. It is also called the multi-quadrics RBF with the expression [24].

$$
\phi_{m q}\left(r_{j}\right)=\sqrt{1+\left(\varepsilon r_{j}\right)^{2}}
$$

where $r_{j}$ is the Euclidean distance between two points. $\varepsilon$ is the RBF shape parameter which can balance the solution accuracy.

As is known to all, a linear combination of RBFs can provide the numerical solutions for boundary value problems governed by elliptic partial differential equations. In literatures, this can be not directly used to solve boundary value problems governed by parabolic or hyperbolic partial differential equations.

To make the direct applications possible, the time variable is treated equally with the space variables. More specifically, we construct a simple direct radial basis function by combining the two-dimensional point $(x, y)$ and the one-dimensional time variable $t$ as a three-dimensional point $(x, y, t)$ for the spacetime domain $\Omega \times t$. For collocation points generation, the time variable is treated equally to the space variables $x$ and $y$. For a simple example, the collocation points in the $x$ - and $y$-directions are evenly chosen with fineness $h_{x}=h_{y}=1 / n$. The time-step for the rest $t$-direction is chosen as $\Delta t=1 / n$.

Similar to the traditional multi-quadric RBF, the extended radial basis function (ERBF) has the form

$\varphi_{m q}\left(r_{j}\right)=\sqrt{1+\varepsilon^{2} r_{j}^{2}}=\sqrt{1+\varepsilon^{2}\left(x-x_{j}\right)^{2}+\varepsilon^{2}\left(y-y_{j}\right)^{2}+\varepsilon^{2}\left(t-t_{j}\right)^{2}}$

where $r_{j}=\left\|P-P_{j}\right\|$ represents the distance between two spacetime coordinates $P=(x, y, t)$ and $P_{j}=\left(x_{j}, y_{j}, t_{j}\right)$.

In literatures [25-27], the other researchers have proposed some space-time radial basis functions with different types. We have done many investigations and found that they are not suitable to deal with the wave equations.

\section{IMPLEMENTATION OF THE EXTENDED RADIAL BASIS FUNCTION}

\subsection{The Extended Radial Basis Function Solution Procedure}

To solve the problem described by Eqs. 1-4, we use the ERBF to present the detailed procedure. Based on the definition of ERBF, 
TABLE 1 | The comparison of the absolute errors at $t=0.5 \mathrm{~s}$.

\begin{tabular}{lccc}
\hline Coordinates & Analytical solutions & ERBF & Error \\
\hline$(0.1,0.1)$ & 0.4145 & 0.4146 & $7.84 \times 10^{-5}$ \\
$(0.3,0.3)$ & -0.1583 & -0.1584 & $6.35 \times 10^{-5}$ \\
$(0.5,0.5)$ & -0.5123 & -0.5123 & $1.90 \times 10^{-5}$ \\
$(0.7,0.7)$ & -0.1583 & -0.1584 & $6.35 \times 10^{-5}$ \\
$(0.9,0.9)$ & 0.4145 & 0.4146 & $7.84 \times 10^{-5}$
\end{tabular}

Eqs 1-4 can be simulated by a one-step numerical method. The unknown function $u(x, y, t)$ can be approximated by the following expression

$$
\bar{u}(\cdot) \approx \sum_{j=1}^{N} \lambda_{j} \varphi_{m q}\left(\cdot_{j}\right),
$$

where the unknown coefficients $\left\{\lambda_{j}\right\}_{j=1}^{n}$ should be solved.

To illustrate the ERBF solution procedure, the internal points $\left\{P_{i}=\left(x_{i}, y_{i}, t_{i}\right)\right\}_{i=1}^{N_{I}}$ are located inside the physical domain and initial boundary points $\left\{P_{i}=\left(x_{i}, y_{i}, t_{i}\right)\right\}_{i=N_{I}+1}^{N_{I}+N_{t}}$ and boundary points $\left\{P_{i}=\left(x_{i}, y_{i}, t_{i}\right)\right\}_{i=N_{I}+N_{t}+1}^{N}$ are located on the physical boundary. By substituting Eq. 7 into Eqs. 1-3, we have

$$
\begin{gathered}
\sum_{j=1}^{N} \lambda_{j} L \varphi_{m q}\left(P_{i}, P_{j}\right)=f\left(P_{i}\right), \quad i=1, \ldots, N_{I}, \\
\sum_{j=1}^{N} \lambda_{j} \varphi_{m q}\left(P_{i}, P_{j}\right)=f_{1}\left(P_{i}\right), \quad i=N_{I}+1, \ldots, N_{I}+N_{t}, \\
\sum_{j=1}^{N} \lambda_{j} \frac{\partial \varphi_{m q}\left(P_{i}, P_{j}\right)}{\partial t}=f_{2}\left(P_{i}\right), \quad i=N_{I}+N_{t}+1, \ldots, N_{I}+2 N_{t}, \\
\sum_{j=1}^{N} \lambda_{j} B \varphi_{m q}\left(P_{i}, P_{j}\right)=f_{3}\left(P_{i}\right), \quad i=N_{I}+2 N_{t}+1, \ldots, N,
\end{gathered}
$$

where

$$
L \varphi_{m q}=\frac{\partial^{2} \varphi_{m q}}{\partial t^{2}}+\alpha \frac{\partial \varphi_{m q}}{\partial t}--\frac{\partial}{\partial t} \nabla \cdot\left(Q \nabla \varphi_{m q}\right)-\nabla \cdot\left(A \nabla \varphi_{m q}\right) .
$$

This is similar with the traditional strong-form collocation approach. We note that the two initial conditions require double initial boundary points, so the total collocation point number is $N=N_{I}+2 N_{t}+N_{b}$.

Eqs. 8-11 has the following matrix form

$$
\mathbf{M} \Lambda=\mathbf{F},
$$

where

$$
\mathbf{M}=\left[\begin{array}{llll}
M_{11} & M_{12} & M_{13} & M_{14} \\
M_{21} & M_{22} & M_{23} & M_{24} \\
M_{31} & M_{32} & M_{33} & M_{34} \\
M_{41} & M_{42} & M_{43} & M_{44}
\end{array}\right]
$$

are $N \times N$ known matrix. $\boldsymbol{\Lambda}$ and $\mathbf{F}$ are $N \times 1$ vectors.

The unknown vector $\Lambda$ in Eq. 13 can be solved by the direct solver in any software codes. Substitute the coefficients into Eq. 7,
TABLE 2 | The comparison of the absolute errors at $t=0.9 \mathrm{~s}$

\begin{tabular}{lccc}
\hline Coordinates & Analytical solutions & ERBF & Error \\
\hline$(0.1,0.1)$ & 1.0177 & 1.0177 & $4.62 \times 10^{-5}$ \\
$(0.3,0.3)$ & -0.3887 & -0.3875 & $1.26 \times 10^{-3}$ \\
$(0.5,0.5)$ & -1.2580 & -1.2593 & $1.28 \times 10^{-3}$ \\
$(0.7,0.7)$ & -0.3887 & -0.3875 & $1.26 \times 10^{-3}$ \\
$(0.9,0.9)$ & 1.0177 & 1.0177 & $4.62 \times 10^{-5}$
\end{tabular}

we get the numerical solution for any points in the whole physical domain.

\subsection{Algorithms of the Extended Radial Basis Function}

For the solution procedure of the ERBF, we summarize the corresponding simple algorithm steps as below.

Step 1. Enter the prescribed functions $f(x, y, t), f_{1}(x, y)$, $f_{2}(x, y), f_{3}(x, y, t)$, and the coefficients $q_{1}, q_{1}, a_{1}, a_{2}$.

Step 2. Select the point parameter $n$ which is connected with the total collocation number $N$.

Step 3. Generate the collocation points $\left(x_{i}, y_{i}, t_{i}\right), I=$ $1,2, \ldots, N$ with internal points $\left\{\left(x_{i}, y_{i}, t_{i}\right)\right\}_{i=1}^{N_{I}}$, initial boundary points $\left\{\left(x_{i}, y_{i}, t_{i}\right)\right\}_{i=N_{I}+1}^{N_{I}+N_{t}}$ and boundary points $\left\{\left(x_{i}, y_{i}, t_{i}\right)\right\}_{i=N_{I}+N_{t}+1}^{N}$.

Step 4. Compute the interpolation matrix $\mathbf{M}$ and the righthand vector $\mathbf{F}$.

Step 5. Solve $\mathbf{M} \boldsymbol{\Lambda}=\mathbf{F}$.

Step 6. Substitute $\boldsymbol{\Lambda}$ into (Eq. 7) to construct the numerical solution $\bar{u}(x, y, t)$.

In this paper, the MATLAB is used to realize the algorithms of ERBF.

\section{NUMERICAL EXPERIMENTS}

In order to study the convergence of ERBF, the relative errors between ERBF and analytical solutions are calculated. It should be noted that the parameter $\varepsilon$ in the ERBF should be chosen first. The optimal choice is similar with the traditional RBFs. For more details about this topic, we refer readers to [24, 28, 29] and references therein. In this paper the parameter is determined with an prior determination. More specifically, the collocation points are fixed first to find the quasi-optimal parameter, after which it will be used for the rest computations.

We consider the problem with $\alpha=1, Q=A=I$, the corresponding exact solution is

$$
u(x, y, t)=\left[1+\frac{1}{2} \sin \left(\pi^{2} t\right)\right] \sin (5 \pi x) \sin (3 \pi y) .
$$

The source function $f(x, y, t)$ can be deduced from the governing Eq. 1 

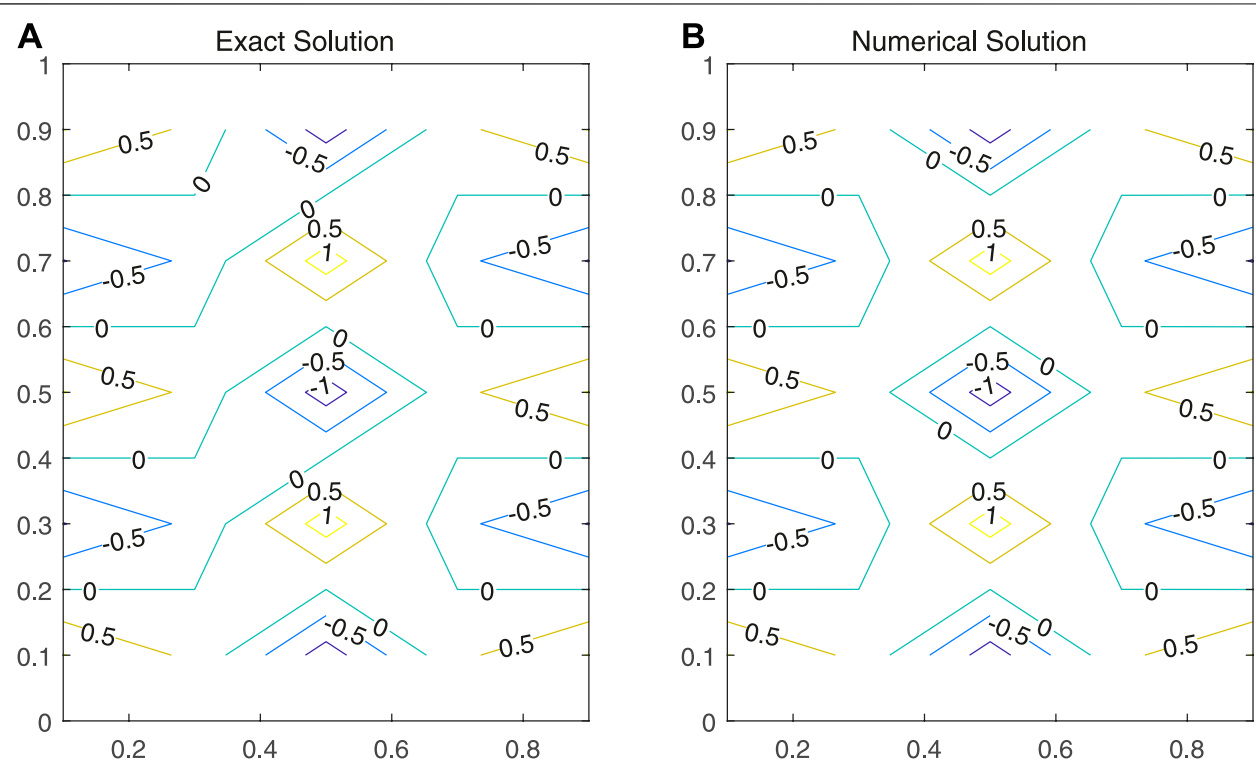

FIGURE 1 | Contour plot of exact solutions (A) and numerical solutions (B).

\begin{tabular}{lccc}
\hline \multicolumn{3}{l}{ TABLE 3 | Maximum absolute error $L^{\infty}$. } & \\
\hline Method & ERBF & Method in [30] & Method in [31] \\
\hline$L^{\infty}$ & $1.28 \times 10^{-3}$ & $4.59 \times 10^{-2}$ & $3.48 \times 10^{-1}$ \\
Time step & $1 / 9$ & 0.02 & 0.02 \\
CPU & $0.92 \mathrm{~s}$ & $15.70 \mathrm{~s}$ & - \\
\hline
\end{tabular}

$$
\begin{gathered}
u(x, y, t)=\pi^{2} \sin (5 \pi x) \sin (3 \pi y)[34+\ldots \\
\left.\left(\frac{1}{2}+17 \pi^{2}\right) \cos \left(\pi^{2} t\right)+\left(17-\frac{1}{2} \pi^{2}\right) \sin \left(\pi^{2} t\right)\right]
\end{gathered}
$$

For fixed collocation point number parameter $n=8$, the total collocation point is 729 , and the number of points in each dimension is $9 \times 9 \times 9$ in $x, y$ and $t$ dimensions, respectively. To reveal the numerical solution accuracy of the proposed method, the wave propagation of nodes is calculated by theoretical formula at $t=0.5 \mathrm{~s}$ and $t=0.9 s$, respectively. From Table 1 and Table 2, we find that numerical solutions of the proposed ERBF are very close with the analytical solutions for different points. The corresponding absolute errors remain the same at $t=0.5 \mathrm{~s}$. And the corresponding absolute errors is a little large for some points, but it is still accurate enough for practical problems.

Figure 1 describes the contour plots of the numerical solutions and analytical solutions at time $t=0.5$. We find that the numerical solutions agree very well with the analytical solutions. It should be noted that the results for the other times have the same accurate results as shown in Figure 1.

Tables 1, 2 show that the numerical results coincide very well with the analytical solutions for both $t=0.5 \mathrm{~s}$ and $t=0.9 \mathrm{~s}$. For time $t=0.5 \mathrm{~s}$, the absolute errors for different points are almost the same. For the time $t=0.9 s$, the absolute errors for points $(0.1,0.1)$ and $(0.9,0.9)$ are more accurate than the other points. Also, it should be pointed out that the ERBF results are more accurate than the other numerical methods [30,31]. The corresponding comparison is revealed in Table 3 by using the maximum absolute error $L^{\infty}$. From which we can see that the time step of the proposed ERBF 1/9 is larger than the other two methods 0.02 , but the corresponding absolute error of the ERBF is smaller than the other two methods. And the computational time of the ERBF $(\mathrm{CPU}=0.92 \mathrm{~s})$ is less than the method $(\mathrm{CPU}=15.70 \mathrm{~s})$ used in [30].

\section{CONCLUSION}

A one-step new general mesh free scheme for solving a viscous wave equation with variable coefficients is proposed. It is based on the strong form-based mesh free collocation method in conjunction with the radial basis functions. Numerical results show that the mesh free method is more simple than the other numerical methods with solution accuracy maintained. The proposed method with the localized technique can be extended to large-scale problems easily.

\section{DATA AVAILABILITY STATEMENT}

The original contributions presented in the study are included in the article/Supplementary Material, further inquiries can be directed to the corresponding author.

\section{AUTHOR CONTRIBUTIONS}

FW designed the whole paper; JZ and IA wrote the manuscript; $\mathrm{AF}$ and HA analyzed experimental results. 


\section{FUNDING}

The work was supported by the Natural Science Foundation of Anhui Province (Project No. 1908085QA09) and the University Natural Science Research Project of Anhui Province (Project No. KJ2019A0591).

\section{REFERENCES}

1. Chen H, Zhou H, Jiang S, and Rao Y. Fractional Laplacian Viscoacoustic Wave Equation Low-Rank Temporal Extrapolation. IEEE Access (2019) 99:1-11. doi:10.1109/ACCESS.2019.2927760

2. Young $\mathrm{DL}, \mathrm{Gu} \mathrm{MH}$, and Fan CM. The Time-Marching Method of Fundamental Solutions for Wave Equations. Eng Anal Bound Elem (2009) 33:1411-25. doi:10.1016/j.enganabound.2009.05.008

3. Wang LH, and Qian ZH. A Meshfree Stabilized Collocation Method (SCM) Based on Reproducing Kernel Approximation November. Comput Method Appl M (2020) 371:113303. doi:10.1016/j.cma.2020.113303

4. Mellouli H, Jrad H, Wali M, and Fakhreddine D. Geometrically Nonlinear Meshfree Analysis of 3D-Shell Structures Based on the Double Directors Shell Theory with Finite Rotations. Steel Compos Struct (2019) 31(4):397-408. doi:10.12989/scs.2019.31.4.397

5. Mellouli H, Jrad H, Wali M, and Dammak F. Meshfree Implementation of the Double Director Shell Model for FGM Shell Structures Analysis. Eng Anal Bound Elem (2019) 99:111-21. doi:10.1016/ j.enganabound.2018.10.013

6. Mellouli H, Jrad H, Wali M, and Dammak F. Free Vibration Analysis of FGCNTRC Shell Structures Using the Meshfree Radial point Interpolation Method. Comput Math Appl (2020) 79(11):3160-78. doi:10.1016/ j.camwa.2020.01.015

7. Wang LH, Liu YJ, Zhou YT, and Yang F. Static and Dynamic Analysis of Thin Functionally Graded Shell with In-Plane Material Inhomogeneity. Int J Mech Sci (2021) 193:106165. doi:10.1016/j.ijmecsci.2020.106165

8. Li PW, and Fan CM. Generalized Finite Difference Method for TwoDimensional Shallow Water Equations. Eng Anal Bound Elem (2017) 80: 58-71. doi:10.1016/j.enganabound.2017.03.012

9. Baccouch M, and Temimi H. A High-Order Space-Time Ultra-weak Discontinuous Galerkin Method for the Second-Order Wave Equation in One Space Dimension. J Comput Appl Math (2021) 389:113331. doi:10.1016/ j.cam.2020.113331

10. Xie X, and Liu YJ. An Adaptive Model Order Reduction Method for Boundary Element-Based Multi-Frequency Acoustic Wave Problems. Comput Method Appl M (2021) 373:113532. doi:10.1016/j.cma.2020.113532

11. Yang SP, Liu FW, Feng LB, and Turner I. A Novel Finite Volume Method for the Nonlinear Two-Sided Space Distributed-Order Diffusion Equation with Variable Coefficients. J Comput Appl Math (2021) 388:113337. doi:10.1016/ j.cam. 2020.113337

12. Takekawa J, and Mikada H, A Mesh-free Finite-Difference Method for Elastic Wave Propagation in the Frequency-Domain. Comput Geosci-uk 118. (2018) p. 65-78. doi:10.1016/j.cageo.2018.05.011

13. Gao LF, and Keyes D. Combining Finite Element and Finite Difference Methods for Iso-Tropic Elastic Wave Simulations in an Energy-Conserving Manner. J Comput Phys (2019) 378:665-85. doi:10.1016/j.jcp.2018.11.031

14. Akers B, Liu T, and Reeger J. A Radial Basis Function Finite Difference Scheme for the Benjamin-Ono Equation. Math (2021) 9(1):65. doi:10.3390/ math9010065

15. Bhardwaj A, and Kumar A. A Meshless Method for Time Fractional Nonlinear Mixed Diffusion and Diffusion-Wave Equation. Appl Numer Math (2021) 160: 146-65. doi:10.1016/j.apnum.2020.09.019

16. Ebrahimijahan A, and Dehghan M. The Numerical Solution of Nonlinear Generalized Benjamin-Bona-Mahony-Burgers and Regularized Long-Wave Equations via the Meshless Method of Integrated Radial Basis Functions. Eng Comput-germany (2021) 37:93-122. doi:10.1007/s00366-019-00811-3

\section{SUPPLEMENTARY MATERIAL}

The Supplementary Material for this article can be found online at: https://www.frontiersin.org/articles/10.3389/fphy.2021.701512/ full\#supplementary-material

17. Rasoulizadeh MN, Nikan O, and Avazzadeh Z. The Impact of LRBF-FD on the Solutions of the Nonlinear Regularized Long Wave Equation. Math Sci (2021) doi:10.1007/s40096-021-00375-8

18. Oruc O. A Radial Basis Function Finite Difference (RBF-FD) Method for Numerical Simulation of Interaction of High and Low Frequency Waves: Zakharov-Rubenchik Equations. Appl Math Comput (2021) 394:125787. doi:10.1016/j.amc.2020.125787

19. Lu X, Zhang P, Shi LW, Hou SM, and Kuang Y. A Radial Basis Function Meshless Numerical Method for Solving Interface Problems in Irregular Domains. Adv Appl Math Mech (2021) 13:645-70. doi:10.4208/aamm.OA-2020-0004

20. Ranocha H, Mitsotakis D, and Ketcheson DI. A Broad Class of Conservative Numerical Methods for Dispersive Wave Equations. Commun Comput Phys (2021) 29:979-1029. doi:10.4208/cicp.OA-2020-0119

21. Netuzhylov H, and Zilian A. Space-time Meshfree Collocation Method: Methodology and Application to Initial-Boundary Value Problems. Int J Numer Meth Eng (2009) 80(3):355-80.

22. Kansa EJ. Multiquadrics-A Scattered Data Approximation Scheme with Applications to Computational Fluid-Dynamics-I. Comput Math Appl (1990) 19(8/9):127-45. doi:10.1016/0898-1221(90)90270-T

23. Karageorghis A, Tappoura D, and Chen CS. The Kansa RBF Method with Auxiliary Boundary Centres for Fourth Order Boundary Value Problems. Math Comput Simulat (2021) 181:581-97. doi:10.1016/j.matcom.2020.10.010

24. Wang FZ, and Hou ER. A Direct Meshless Method for Solving TwoDimensional Second-Order Hyperbolic Telegraph Equations. J Math-uk (2020) 8832197. doi:10.1155/2020/8832197

25. Myers DE, Iaco SD, Posa D, and Cesare LD. Space-time Radial Basis Functions. Comput Math Appl (2002) 43:539-49. doi:10.1016/S0898-1221(01)00304-2

26. Wang FZ, Hou ER, Ahmad I, and Ahmad H. An Efficient Meshelss Method for Hyperbolic Telegraph Equations. Cmes-comput Model Eng (2021) doi:10.32604/cmes.2021.014739

27. Wang FZ, Zheng KH, Ahmad I, and Ahmad H. Gaussian Radial Basis Functions for Linear and Nonlinear Convection-Diffusion Problems. Open Phys (2021) 19:69-76. doi:10.1515/phys-2021-0011

28. Rippa S. An Algorithm for Selecting a Good Value for the Parameter C in Radial Basis Function Interpolation. Adv Comput Math (1999) 19:193-210. doi:10.1023/A:1018975909870

29. Fasshauer GE, and Zhang JG. On Choosing Optimal Shape Parameters for RBF Approximation. Numer Algorithms (2007) 45:345-68. doi:10.1007/ s11075-007-9072-8

30. Su MY, Ren ZH, and Zhang ZY. An ADI Finite Volume Element Method for a Viscous Wave Equation with Variable Coefficients. Cmes-comp Model Eng (2020) 123(2):739-76. doi:10.32604/cmes.2020.08563

31. Zampieri E, and Pavarino LF. Implicit Spectral Element Methods and Neumann-Neumann Preconditioners for Acoustic Waves. Comput Method Appl M (2006) 195(19-22):2649-73. doi:10.1016/j.cma.2005.06.005

Conflict of Interest: The authors declare that the research was conducted in the absence of any commercial or financial relationships that could be construed as a potential conflict of interest.

Copyright (c) 2021 Wang, Zhang, Ahmad, Farooq and Ahmad. This is an open-access article distributed under the terms of the Creative Commons Attribution License (CC $B Y)$. The use, distribution or reproduction in other forums is permitted, provided the original author(s) and the copyright owner(s) are credited and that the original publication in this journal is cited, in accordance with accepted academic practice. No use, distribution or reproduction is permitted which does not comply with these terms. 